\title{
Cuantificación de hongos micorrícicos en muestras de suelo en plantaciones de Tabebuia rosea y Cordia alliodora
}

\author{
Cuervo A. Jairo L'1, Rivas P. Gonzalo G. ${ }^{2}$ \\ 'Docente investigador Universidad Nacional de Colombia \\ ${ }^{2}$ Coordinador Grupo Agroecología del Centro Agronómico Tropical de Investigación y Enseñanza CATIE \\ Correspondencia: jlcuervoa@unal.edu.co \\ Recibido: 16-045-2007 / Aceptado: 08-05-2007
}

\begin{abstract}
Resumen
Después de que han sido intervenidos y sobreexplotados por el hombre; los suelos tropicales presentan grandes limitantes para la producción de árboles. Una estrategia para aumentar los niveles de producción se basa en la fertilización acorde con los requerimientos de los árboles. Como la micorrización es una alternativa biológica de bajo costo, que puede contribuir al éxito de la repoblación forestal, se quiso establecer cómo se podría favorecer la sobrevivencia y el crecimiento de especies arbóreas en viveros por medio de la cuantificación de hongos micorricicos utilizando muestras de suelo en las especies Tabebuia rosea y Cordia alliodora en diferentes localidades de Costa Rica, Centro América.

Se analizaron muestras en diez sitios diferentes. La cuantificación de esporas se realizó mediante la técnica de tamizado y el porcentaje de colonización de raíces se estimó mediante la observación microscópica de raíces según metodología de Sieverding. Los resultados obtenidos en esta investigación permitieron concluir que, de la variedad de los géneros de Micorrizas Vesiculo Arbusculares presentes en la muestra de suelo, los que demostraron mejor comportamiento con Tabebuia rosea y Cordia. alliodora fueron Glomus sp y Gigaspora sp. Además para lograr un mayor crecimiento y desarrollo de los árboles, es necesario favorecer la población micorrizal en las plantas en sus primeros días de nacidas.
\end{abstract}

Palabras clave: biofertilización, Cordia alliodora, micorrización, reforestación, Tabebuia rosea

\begin{abstract}
The tropical grounds show great restrictions for tree production after they have been used and over-exploited by man. A strategy to increase the levels of production is based on the fertilization according to the requirements of the trees. As mycorrhization is a biological low cost alternative that can contribute to the success of the reforestation, we wanted to establish how it was possible to favor the surviving and growth of arboreal species in green houses by means of the quantification of mycorrhizal fungi using ground samples in the species Tabebuia rosea and Cordia alliodora in different localities from Costa Rica, Central America.
\end{abstract}


Samples in ten different sites were analyzed and the quantification of spores was made by sifting. The percentage of colonization by roots was considered at microscopic observation by roots according to methodology of Sieverding. The results obtained in this investigation concluded that from the variety of the present sorts of Micorrizas Vesiculo Arbusculares in the ground sample those that demonstrated better behavior with Tabebuia rosea and Cordia alliodora were Glomus sp. and Gigaspora sp. In addition, to obtain a greater growth and development of the trees, it is necessary to favor the mycorrhizal population in the plants within their first days of birth.

Key words: biofertilization, Cordia alliodora, mycorrhization, reforestation, Tabebuia rosea.

\section{Introducción}

Debido a la carencia aguda de fósforo en la mayor parte de sus suelos, en el trópico el estudio de la simbiosis micorriza-planta adquiere particular importancia. Se ha demostrado que la selección de cepas de hongos Micorrizas Vesiculo Arbusculares (MVA) eficientes y su producción masiva a escala industrial pueden contribuir a un gran beneficio para el éxito de la repoblación forestal, demostrándose que la simbiosis de MVA constituye una valiosa ayuda para mejorar tanto el crecimiento de pasturas de distintos forestales como el balance ecológico de un ecosistema $(1,2)$.

Desde una perspectiva práctica los hongos micorrizógenos son más que biofertilizantes. Aunque el principal beneficio es de carácter nutricional, una planta puede obtener ventajas adicionales de los hongos micorrícicos, como tolerancia a estrés hídrico, tolerancia a salinidad y protección a enfermedades, entre otros (3).

Los hongos vesículo-arbusculares (VA), además de su efecto directo en la nutrición de las plantas, inducen cambios fisiológicos que comprenden un aumento en la tasa fotosintética y redistribución del carbono fijado en mayor proporción hacia las raíces $(4,5)$. En la planta ocurren cambios en la concentración y proporción de hormonas que se manifiestan en la prolongación del ciclo vegetativo e incide principalmente en la floración de esta.

La manipulación de microorganismos para el manejo de los suelos forestales tiene un gran potencial. Una vez las técnicas para su utilización y manejo estén bien desarrolladas, se podría reducir los esfuerzos y costos de la reforestación, manteniendo los óptimos niveles de nitrógeno y materia orgánica y mejorando la capacidad de captación del fósforo. Además de establecer manejos adecuados de los árboles y su habilidad para soportar el estrés por falta de agua y nutrientes, e incrementar la productividad de los suelos (6).

Motivados por el papel que desempeñan las micorrizas como promotoras del crecimiento, se pretende mostrar una metodología que permita la manipulación de estos microorganismos nativos, que facilite trabajos futuros para la siembra y establecimiento de árboles. Para ello, se realizó el conteo de esporas y porcentaje de colonización de los hongos micorricícos en 20 muestras de suelos, que se obtuvieron de diferentes localidades en Costa Rica, Centro América, en donde se encontraba creciendo las especies forestales Tabebuia rosea y Cordia alliodora, con el fin de conocer la población micorrizal para estas dos especies.

\section{Materiales y Métodos}

\section{Muestreo}

Se localizaron 10 sitios en diferentes localidades de Costa Rica (Tabla 1), donde se encontraba creciendo Tabebuia rosea y Cordia alliodora, procurando que los sitios estuvieran en el rango ecológico de adaptación reportados para las dos especies forestales. Por cada sitio se muestreo de 6-8 árboles, tomando 2 submuestras de suelo por árbol. Para la toma de muestras se procedió a sacar suelo con un barreno, a $25 \mathrm{~cm}$ de profundidad, las cuales se realizaron en el área de crecimiento radical del árbol (proyección de la copa sobre el suelo) y en la 
parte intermedia (entre el borde del área de crecimiento y el tronco del árbol). Las submuestras de cada sitio se mezclaron hasta obtener $2 \mathrm{~kg}$ de suelo por sitio.

\section{Procedimiento}

Mediante la técnica de tamizado sugerida por Brundrett, las esporas de MVA se extrajeron y cuantificaron a partir de una muestra de $100 \mathrm{~g}$ de suelo (7). El porcentaje de colonización de raíces se estimó mediante la observación microscópica (40X) de raíces clareadas con $\mathrm{KOH}$ al 10\% y teñidas con azul de tripano al $0.05 \%$ según la metodología sugerida por Sieverding (8). Los datos obtenidos se corrieron con el sistema estadístico SAS.

Tabla 1. Localidades de Costa Rica, Centro América, donde se efectuaron los muestreos.

\begin{tabular}{ll}
\hline LOCALIDAD & CODIGO \\
\hline San José & SJ \\
Cartago & CTG \\
Alajuela & ALA \\
\hline
\end{tabular}

\section{Resultados y discusión}

Las pruebas de ANOVA obtenidas por análisis estadístico para las variables, número de especies MVA, porcentaje de colonización y número de esporas no presentó valores estadísticamente significativos ( $\mathrm{p}<0.01)$, lo que nos hace pensar que la población micorrizal es muy importante para estas dos especies forestales. De ser así, se hace necesario favorecer la población micorrizal en las plantas, en sus primeros días de nacidas, para lograr un mayor crecimiento y desarrollo de los árboles.

Los géneros más abundantes de hongos MVA encontrados en las muestras de suelo, para las especies forestales Tabebuia rosea y Cordia alliodora fueron Glomus spp. y Gigaspora spp.; con $60 \%$ y $20 \%$ respectivamente. Otros hongos MVA no se identificaron taxonómicamente. Castaño, citado por Ospina y Martínez $(9,10)$ reportaron la presencia de Glomus manihotis en Cordia. alliodora y Tabebuia rosea. El número de especies de MVA presentes en las muestras de suelo, demuestra una variedad de estos microorganismos interactuando con estas especies forestales, Figuras 1 y 2.

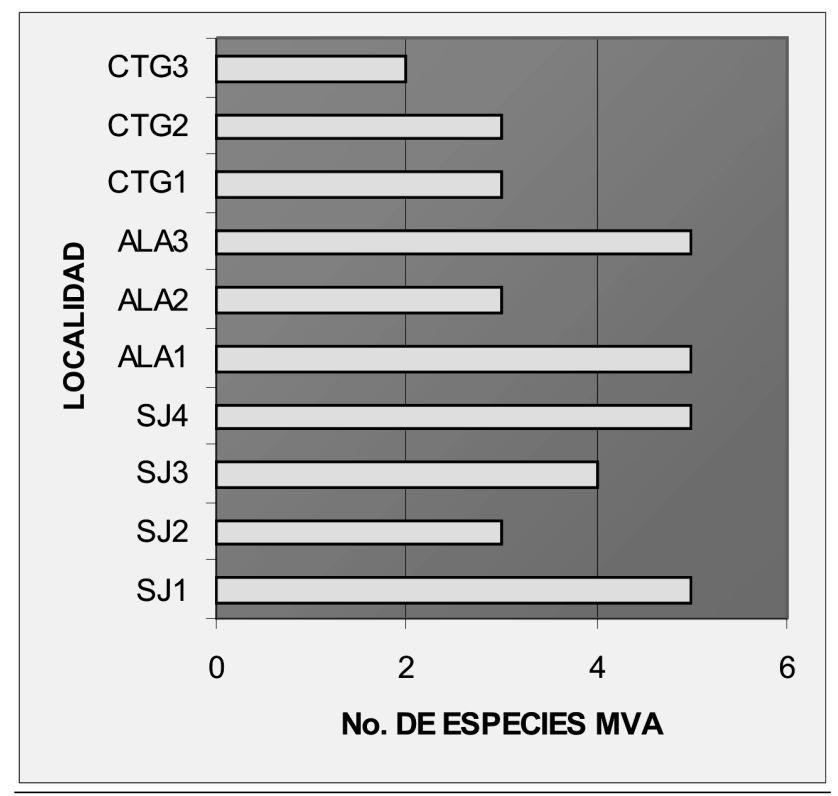

Figura 1. Número de especies MVA / localidad, asociadas con $T$. rosea en Costa Rica (CTG= Cartago; ALA= Alajuela y SJ= San José).

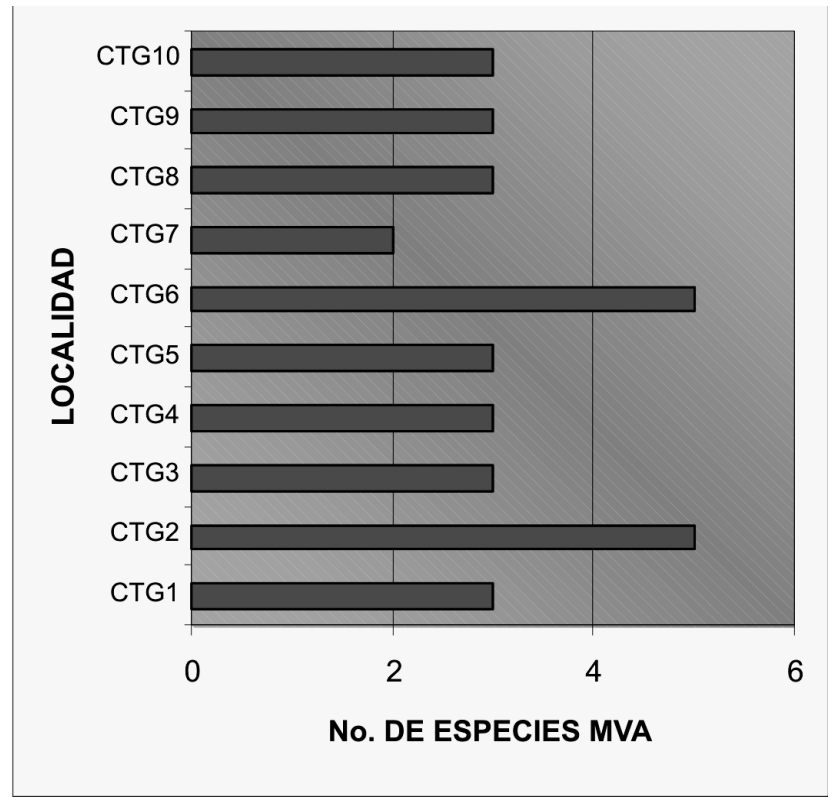

Figura 2. Número de especies MVA / localidad, asociadas con $C$. alliodora en Costa Rica (CTG= Cartago). 


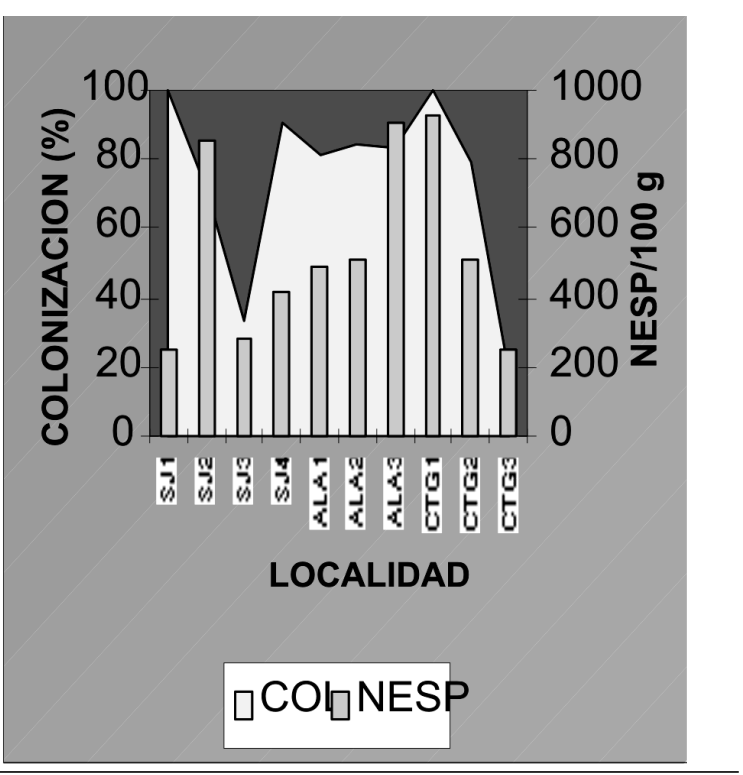

Figura 3. Colonización (\%) y Número de esporas de MVA/sitio asociada con T.rosea (CTG= Cartago; $\mathrm{ALA}=$ Alajuela y $\mathrm{SJ}=\mathrm{San}$ José).

En cuanto al número de esporas y el porcentaje de colonización, se encontró diferencias significativas entre especies, siendo Cordia alliodora la que presentó el mayor número, con un promedio de 558/100 g de suelo; para Tabebuia rosea fue de 514/100 g de suelo.

El porcentaje de colonización, para las dos especies, no presentó correlación con el número de esporas, lo cual indica que una alta colonización no está siempre relacionada con un alto número de esporas, Figuras 3 y 4. Según Miranda el número de esporas en el suelo y el porcentaje de colonización de la raíz puede ser considerado como un indicativo del establecimiento de los hongos en el suelo, más que un indicativo de la efectividad en el crecimiento de las plantas (11).

A manera de conclusión, este estudio permite resaltar y demostrar la importancia de las micorrizas y su potenciación en las plantaciones, ya que su uso tiene varios resultados positivos, entre ellos, una menor necesidad de fertilización con químicos, potenciación de los procesos de desarrollo arbuscular y disminución de costos. Además, a mediano plazo, aumenta también la posibilidad de mejorar la producción.

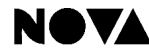

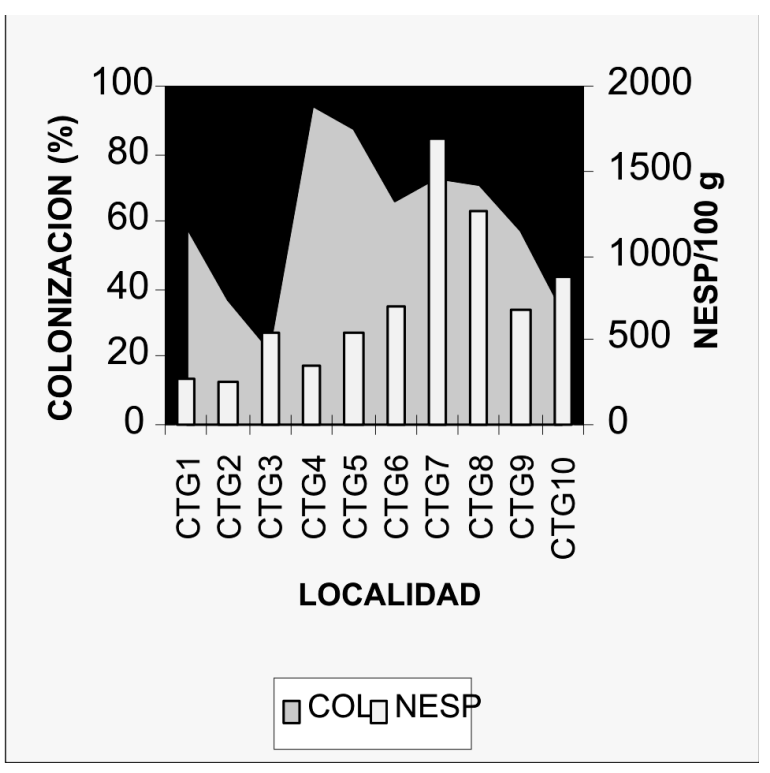

Figura 4. Colonización (\%) y número de esporas de MVA/sitio asociada con $C$. alliodora $(\mathrm{CTG}=$ Cartago).

\section{Referencias}

1. Kernaghan G. Mycorrhizal diversity: Cause and effect?. Pedobiologia. 2005;49:511-520.

2. Patiño H, Quintero H. Trascendencia ecológica de la selva tropical con referencia especial al neotrópico. Partes I y II. Rev. Coagro. 1982; 38 y 39.

3. Barea J, Andrade G, Bianciotto V, Dowling D, Lohrke S, Bonfante P, O'Gara F, Azcon-Aguilar C. Impact on arbuscular mycorrhiza formation of pseudomonas strains used as inoculants for biocontrol of soil-borne fungal plant pathogens. Appl Environ Microbiol. 1998;64:2304-2307.

4. Azcon R. Papel de la simbiosis micorrizica y su interacción con otros microorganismos rizosféricos en el crecimiento vegetal y sostenibilidad agrícola. In Alarcón A, Ferrera-Serraro R. Ecología, fisiología y biotecnología de la micorriza arbuscular. Ed. Mundi-Prensa México. 2000.

5. Blanco F, Salas E. Micorrizas en la agricultura: contexto mundial e investigación realizada en Costa Rica. In: Congreso Nacional Agronómico y de Recursos Naturales, Congreso Nacional de Fitopatología, Congreso Nacional de suelos (2, 1996, San José, C. R) ¿Puede la agricultura sostenible ser competitiva?: Memoria. Eds. J. Bertseh; W. Badilla; E. Bornemiza. San José, EUNED/EUNA. 1996;3:69-79.

6. Fortin J, Carlisle A. The use of root symbiosis in intensive forestry. In. Biomass Growth and Production and ENFOR. Environment Canada. Canadian forestry service. Quebec, Canada. Report No. 4. 1984:96.

7. Brundrett M, Melville L, Peterson L. Practical methods in mycorrhiza research. Canada. Mycologie Publications. 1994; 161.

8. Sieverding E. Manual de métodos para las de la micorriza vesículo-arbuscular. CIAT. 1983. Cali, Colombia.

9. Ospina A, Martínez F. La micorriza, un milagro como biofertilizante en cultivos agrícolas y forestales en Colombia. Agronomía colombiana. 1993;5:67-79.

10. Guerrero E. Micorriza: Fundamentos biológicos y estado del arte. In: Micorrizas Recurso Biológico del Suelo. Fondo FEN, Colombia. 1996:1-46.

11. Miranda J. Influencia de hongos micorrizógenos inoculados en el campo, cultivo de sorgo e soja en un solo sobcerrado. Bras. Ci. Solo. 1982;6:19-23 